\title{
A SCHWARZ LEMMA FOR CANONICAL ALGEBRAIC MANIFOLDS
}

\author{
MYUNG H. KWACK
}

\begin{abstract}
It was proved that a holomorphic mapping with a fixed point between a canonical algebraic manifold is biholomorphic if and only if the absolute value of the determinant of its differential at the fixed point is 1 .
\end{abstract}

In this note we prove the following theorem.

THEOREM. Let $V_{n}$ be a compact complex manifold such that the canonical line bundle $K=\wedge^{n} T_{V}^{*}$ is very ample $\left(n=\operatorname{dim}_{C} V\right)$. Let $x_{0}$ be a point of $V$ and $f: V \rightarrow V$ be a holomorphic mapping such that $f\left(x_{0}\right)=x_{0}$. We denote by $d f_{0}: T_{0}(V) \rightarrow T_{0}(V)$ the differential of $f$ at $x_{0}$ where $T_{0}(V)$ denotes the tangent space of $V$ at $x_{0}$. Then

$$
\left|\operatorname{det} d f_{0}\right| \leqq 1,
$$

$$
\begin{aligned}
& d f_{0} \text { is the identity transformation if and only } \\
& \text { if } f \text { is the identity transformation of } V \text {, and }
\end{aligned}
$$

$$
\left|\operatorname{det} d f_{0}\right|=1 \text { if and only if } f \text { is a biholomorphic mapping. }
$$

For bounded domain and hyperbolic manifold, the above theorem is due to H. Cartan and S. Kobayashi [2].

Before we start proving the theorem we recall a few facts, proved by Griffiths [1], about holomorphic mappings into a canonical algebraic manifold (i.e. $\wedge^{n} T_{V}^{*}$ is very ample).

Proposition 1. Let $V$ be a complete canonical algebraic manifold of dimension $n$ and $w_{0}, \cdots, w_{N}$ be a basis for the vector space of holomorphic $n$-forms on $V$. Suppose that $\left\{f_{k}^{\#}\right\}$ is an arbitrary sequence of holomorphic mappings of the unit ball $B$ about 0 of $C^{n}$ into $V$. Then

(1) There exists a subsequence $\left\{f_{k}\right\}$ of $\left\{f_{k}^{\#}\right\}$ such that the pull-backs $f_{k}^{*} w_{i}$ converge uniformly on compact sets to a holomorphic n-form $\varphi_{i}$ $(i=0, \cdots, N)$.

Received by the editors March 2, 1973.

AMS (MOS) subject classifications (1970). Primary 32A10, 32A30.

(c) American Mathematical Society 1973 
(2) If $\mid$ det $d f_{k}(0) \mid \geqq 1$, the meromorphic mapping $f: B \rightarrow V$ given by the homogeneous coordinates $f=\left[\varphi_{0}, \cdots, \varphi_{N}\right]$ is holomorphic in a neighborhood $U$ of 0 and the sequence $\left\{f_{k}\right\}$ converges uniformly on compact subsets of $U$ to $f$.

Proposition 2. Let $V$ be a complete canonical algebraic manifold of dimension $n$ and $B$ the unit ball about 0 in $C^{n}$. Let $f: B \rightarrow V$ be an arbitrary holomorphic mapping with $|\operatorname{det} d f(0)| \geqq 1$. Then there exists an absolute constant $r>0$ such that there is a univalent ball $\Delta(f(0), r)$ of radius $r$ about $f(0)$ for $f: B \rightarrow V$.

A univalent ball $\Delta\left(x_{0}, r\right)$ for $f: B \rightarrow V$ is by definition a disc $\Delta\left(x_{0}, r\right)$ on $V$ of radius $r$ about $x_{0}$ such that $f$ maps some open set $U$ in $B$ biholomorphically onto $\Delta\left(x_{0}, r\right)$. The above two propositions are proved in [1].

Now we prove our theorem. Let $f: V \rightarrow V$ be a holomorphic mapping such that $f\left(x_{0}\right)=x_{0}$. Assume (1) of the theorem is false, i.e. $\mid$ det $d f_{0} \mid=a>1$. For each positive integer $k$, the mapping $f^{k}=f \circ \cdots \circ f(k$-times) satisfies $\left|\operatorname{det} d f_{0}^{k}\right|=a^{k}$. By Proposition 1, there is a neighborhood $U$ of $x_{0}$ such that, when restricted to $U$, a subsequence of $\left\{f^{k}\right\}$ converges uniformly on compact subsets of $U$ to a holomorphic mapping $g: U \rightarrow V$. Since $a^{k}$ diverges to infinity as $k$ goes to infinity, we arrive at a contradiction. This proves (1).

We denote by $d^{m} f_{0}$ all partial derivatives of $f$ of order $m$ at $x_{0}$. We will show that if $d f_{0}$ is the identity transformation of $T_{0}(V)$, then $d^{m} f_{0}=0$ for $m \geqq 2$. Let $m$ be the least integer $\geqq 2$ such that $d^{m} f_{0} \neq 0$. Then $d^{m}\left(f^{k}\right)_{0}=$ $k d^{m} f_{0} \neq 0$ for all positive integers $k$. As $k$ goes to infinity, $d^{m}\left(f^{k}\right)_{0}$ also goes to infinity in contradiction to the fact that a subsequence of $\left\{f^{k}\right\}$ converges uniformly to a holomorphic mapping in a neighborhood of $x_{0}$. This proves (2).

If $f$ is a biholomorphic mapping, then the inverse $f^{-1}$ is a well-defined holomorphic mapping of $V$ into $V$ such that $f^{-1}\left(x_{0}\right)=x_{0}$. Therefore if $\left|\operatorname{det} d f_{0}\right|<1$, $\left|\operatorname{det} d f_{0}^{-1}\right|>1$. By (1) of the theorem we must have $\left|\operatorname{det} d f_{0}\right|=1$ if $f$ is a biholomorphic mapping.

Assume $\mid$ det $d f_{0} \mid=1$. The mapping $f^{k}=f \circ \cdots \circ f$ ( $k$-times) has a subsequence which converges uniformly on compact subsets to a holomorphic mapping $g$ is a neighborhood of $x_{0}$. Let $\lambda$ denote an eigenvalue of $d f_{0}$. Then $d f_{0}^{k}$ has an eigenvalue $\lambda^{k}$. If $|\lambda|>1$, then $\lambda^{k}$ goes to infinity as $k$ goes to infinity, which is a contradiction. Therefore the eigenvalues of $d f_{0}$ have absolute value less than or equal to one. As $\left|\operatorname{det} d f_{0}\right|=1,|\lambda|=1$. Now put $d f_{0}$ in Jordan canonical form. We claim that $d f_{0}$ is then in diagonal form, and the diagonal entries are all of the form $e^{i \theta}$. If it is not, it must 
have a diagonal block of the form

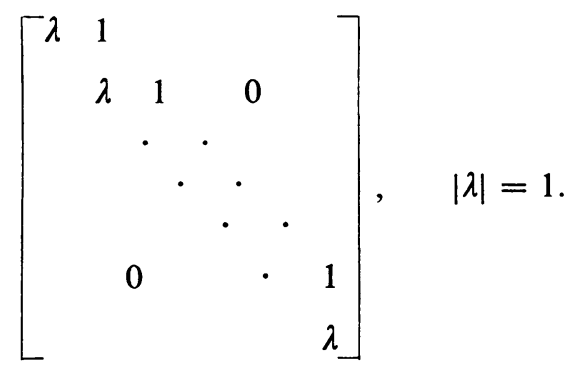

The corresponding block of $d f_{0}^{k}$ is then of the form

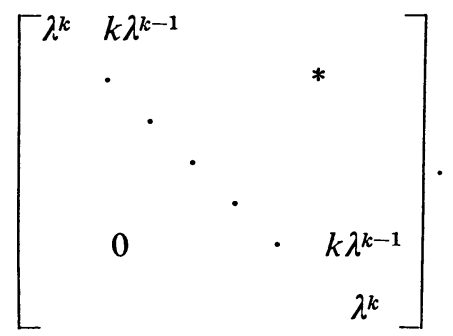

It follows that the entries $k \lambda^{k-1}$ immediately above the diagonal of $d f_{0}^{k}$ diverge to infinity as $k$ goes to infinity. This is again a contradiction to the convergence of a subsequence of $\left\{f^{k}\right\}$ in a neighborhood of $x_{0}$.

Since $d f_{0}$ is a diagonal matrix whose entries have absolute value 1 , there is a subsequence $\left\{d f_{0}^{k(i)}\right\}$ of $\left\{d f_{0}^{k}\right\}$ such that $\left\{d f_{0}^{k(i)}\right\}$ converges to the identity matrix. By Proposition 1, there is a subsequence of $\left\{f^{k(i)}\right\}$, denoted again by $\left\{f^{k(i)}\right\}$, which converges uniformly to a holomorphic mapping $h$ on a compact neighborhood of $x_{0}$. Since $\left\{d f_{0}^{k(i)}\right\}$ converges to $d h_{0}, d h_{0}$ is the identity matrix. Then $h$ is the identity mapping of a neighborhood of $x_{0}$, which can be proved in a similar way as (2) of the theorem.

Let $W$ be the largest open subset of $V$ with the property that some subsequence of $\left\{f^{k(i)}\right\}$ converges to the identity transformation of $W$ uniformly on compact subsets. Without loss of generality we may assume that $\left\{f^{k(i)}\right\}$ converges to the identity transformation of $W$ uniformly on compact subsets. Let $p \in \bar{W}$ and $U$ be a neighborhood of $p$ with compact closure. By Proposition 1, there is a subsequence of $\left\{f^{k(i)}\right\}$, denoted again by $\left\{f^{k(i)}\right\}$, such that the pull-backs $f^{k(i) *}\left(w_{j}\right)(j=0, \cdots, N)$ converge uniformly on compact subsets to holomorphic $n$-forms $\varphi_{j}$, where $w_{0}, \cdots, w_{N}$ are a basis for the vector space of holomorphic $n$-forms on $V$. Then the mapping given by the homogeneous coordinates $f=\left[\varphi_{0}, \cdots, \varphi_{N}\right]$ is the identity mapping on $W \cap U$ and is meromorphic on $U$. It follows that 
$f=\left[\varphi_{0}, \cdots, \varphi_{N}\right]$ must define the identity mapping on $U$. This proves that $W$ is closed and hence $W=V$.

By change of notation we may assume that $\left\{f^{k(i)}\right\}$ converges to the identity transformation of $V$ uniformly on compact subsets. To show $f$ is one-toone, let $f(x)=f(y)$. Then $f^{k(i)}(x)=f^{k(i)}(y)$ and as $k(i)$ goes to infinity, we get $x=y$. This shows $f$ is one-to-one. Finally we show $f$ is onto. Suppose it is not. Then there is a point $p$ in $V-f(V)$. We also have $p \in V-f^{k(i)}(V)$ for all $k(i)$. Since $\left\{d f^{k(i)}\right\}$ converges to the identity matrix, $\left|\operatorname{det} d f_{p}^{k(i)}\right| \geqq 1-$ $\varepsilon>0$ for sufficiently large $k(i)$ 's. By Proposition 2, there is a univalent ball for $f^{k(i)}$ about $f^{k(i)}(p)$ of radius $r>0$ for sufficiently large $k(i)$ 's. Since $f^{k(i)}(p)$ converges to $p$ as $k(i)$ goes to infinity, $p$ belongs to all of these univalent balls for all sufficiently large $k(i)$ 's. Hence $p \in f^{k(i)}(V)$ for all sufficiently large $k(i)$ 's contradicting $p \in V-f^{k(i)}(V)$. This proves the theorem.

\section{REFERENCES}

1. P. Griffiths, Holomorphic mappings into canonical algebraic varieties, Ann. of Math. (2) 93 (1971), 439-458. MR 43 \#7668.

2. S. Kobayashi, Hyperbolic manifolds and holomorphic mappings, Dekker, New York, 1970. MR 43 \#3503.

3. H. Wu, Normal families of holomorphic mappings, Acta Math. 119 (1967), 193-233. MR 37 \#468.

Department of Mathematics, Howard University, Washington, D.C. 20001 